Article

\title{
Favored Inflationary Models by Scalar Field Condensate Baryogenesis
}

\author{
Daniela Kirilova *D and Mariana Panayotova \\ Institute of Astronomy with NAO Rozhen, Bulgarian Academy of Sciences, 72 Tsarigradsko shose Blvd., \\ 1784 Sofia, Bulgaria; mariana@astro.bas.bg \\ * Correspondence: dani@astro.bas.bg; Tel.: +359-886-83-11-38
}

Citation: Kirilova, D.; Panayotova, M. Favored Inflationary Models by Scalar Field Condensate Baryogenesis. Galaxies 2021, 9, 49. https://doi.org/ $10.3390 /$ galaxies 9030049

Academic Editor: Emilio Elizalde

Received: 26 May 2021

Accepted: 6 July 2021

Published: 10 July 2021

Publisher's Note: MDPI stays neutral with regard to jurisdictional claims in published maps and institutional affiliations.

Copyright: (c) 2021 by the authors. Licensee MDPI, Basel, Switzerland. This article is an open access article distributed under the terms and conditions of the Creative Commons Attribution (CC BY) license (https:/ / creativecommons.org/licenses/by/ $4.0 /)$.

\begin{abstract}
We calculate the baryon asymmetry value generated in the Scalar Field Condensate (SCF) baryogenesis model obtained in several inflationary scenarios and different reheating models. We provide analysis of the baryon asymmetry value obtained for more than 70 sets of parameters of the SCF model and the following inflationary scenarios, namely: new inflation, chaotic inflation, Starobinsky inflation, MSSM inflation, quintessential inflation. We considered both cases of efficient thermalization after inflation and delayed thermalization. We have found that the SFC baryogenesis model produces baryon asymmetry orders of magnitude bigger than the observed one for the following inflationary models: new inflation, new inflation model by Shafi and Vilenkin, MSSM inflation, chaotic inflation with high reheating temperature and the simplest Shafi-Vilenkin chaotic inflationary model. Strong diluting mechanisms are needed for these models to reduce the resultant baryon excess at low energies to its observational value today. We have found that a successful generation of the observed baryon asymmetry is possible by the SCF baryogenesis model in Modified Starobinsky inflation, chaotic inflation with low reheating temperature, chaotic inflation in SUGRA, and Quintessential inflation.
\end{abstract}

Keywords: early universe; models of inflation; SFC baryogenesis; reheating

\section{Introduction}

The inflationary paradigm for the description of the very early Universe is already more than 30 years old; however, there still exist numerous models of inflation [1]. Moreover, the reheating process at the end of inflation that is believed to have provided the transfer of the energy stored in the inflaton to other fields and thus enabled the beginning of the radiation dominated stage of the Universe, could have also proceeded through different mechanisms (perturbative [2], nonperturbative [3] (see also the discussion of different reheating mechanisms in ref. [4])). Moreover, different decay channels and different decay rates of the inflaton and other particles, and different thermalization models (instant or delayed) are possible [5]. Therefore, it is interesting to consider the possibility for production of the observed baryon asymmetry $\beta \sim 6 \times 10^{-10}$ for different reheating temperatures in different inflationary models.

In this work we analyze the baryon asymmetry generation according to the Scalar Field Condensate (SFC) baryogenesis model for several inflationary scenarios and different reheating models.

\section{Baryon Asymmetry of the Universe}

The generation of the baryon asymmetry of the Universe is one of the open cosmological issues. Both cosmic ray data and gamma ray data indicate that there are no significant quantities of antimatter in the local vicinity up to galaxy cluster scales of 10-20 Mpc [6-10]. It is most probable that our universe is made of matter. 
The baryon asymmetry is usually described by:

$$
\beta=\left(N_{b}-N_{\bar{b}}\right) / N_{\gamma} \sim N_{b} / N_{\gamma}=\eta
$$

where $N_{b}$ is the number of baryons, $N_{\bar{b}}$ is the number of anti-baryons, $N_{\gamma}$ - the number of photons.

The baryon-to-photon ratio $\eta$ is precisely measured today, namely:

$$
\eta \sim 6 \times 10^{-10}
$$

the best baryometers being BBN and CMB measurements. Deuterium is the most sensitive to $\eta$ among the light elements produced during BBN. Thus, the most precisely obtained $\eta$, based on BBN theory and D observations is [11]:

$$
\eta_{D}=6 \pm 0.3 \times 10^{-10} \text { at } 95 \% \text { C.L. }
$$

The CMB anisotropy data measures $\eta$ with comparative accuracy, namely (see ref. [12]):

$$
\eta_{C M B}=6.11 \pm 0.04 \times 10^{-10} \text { at } 68 \% \text { C.L. }
$$

Although these independent measurements correspond to quite distant epochs, namely BBN proceeds at $z \sim 10^{9}$, while CMB at $z \sim 1000$ they are in excellent agreement, i.e., there was no change in this ratio between the two epochs.

At present there exist many baryogenesis models which successfully generate this number at quite different epochs: in the wide range between the end of inflation and before BBN. Just to mention the most popular ones: GUT baryogenesis [13,14], SUSSY baryogenesis, baryogenesis through leptogenesis [15], Afleck and Dine baryogenesis [16], Scalar Field Condensate baryogenesis (SFC) [2,17], warm baryogenesis [18], Gravitational baryogenesis in Einstein's gravity [19] and in modified gravity theories [20-22], etc.

In the next section we provide a short description of the SFC baryogenesis model. In the third section we present our results for the calculated baryon asymmetry $\beta$ in different inflationary scenarios and different reheating models. In the conclusion we list the main results and present a short discussion.

\section{SFC Short Description}

First ideas on the SFC baryogenesis model and the analytical construction of that model were presented in Refs. [2,17]. In following publications an inhomogeneous SFC baryogenesis model was explored semi-analytically, and was applied to explain the very large scale structure in the universe and the quasi-periodicity found at very large scales with typical period of $128 h^{-2} \mathrm{Mpc}$ [23-25]. Since the first analytical considerations [2] it is known that particle creation processes play an important role for the determination of the baryon asymmetry generation in that model. Recently, a more precise numerical account of particle creation processes and their role in SFC baryogenesis was provided in Refs. [26-28].

According to theSCF baryogenesis model at the inflationary stage there existed the inflaton $\psi$ and a complex scalar field $\varphi$, carrying baryon charge. During inflation, as a result of the rise of quantum fluctuations of $\varphi$, a condensate $\langle\varphi\rangle \neq 0$ with a nonzero baryon charge $B$ is formed [29-31]. $B$ is not conserved at large $\varphi$ due to the presence of $B$ non-conserving (BV) self-interaction terms in the potential $\mathrm{V}(\varphi)$.

The equation of motion of $\varphi$ is:

$$
\ddot{\varphi}+3 H \dot{\varphi}+\frac{1}{4} \Gamma_{\varphi} \dot{\varphi}+U_{\varphi}^{\prime}=0
$$

where $a(t)$ is the scale factor, $H$ is the Hubble parameter $H=\dot{a} / a . \Gamma_{\varphi}=\alpha \Omega$ is the rate of particle creation, $\Omega=2 \pi / T$, where $\mathrm{T}$ is the period of the field oscillations. The analytically estimated value: $\Omega_{0}=\lambda^{1 / 2} \varphi_{0}$, is used as an initial condition for the frequency. 
The baryon charge is given by:

$$
B=-i\left(\dot{\varphi}^{*} \varphi-\dot{\varphi} \varphi^{*}\right) .
$$

The potential is chosen of the form:

$$
U(\varphi)=m^{2} \varphi^{2}+\frac{\lambda_{1}}{2}|\varphi|^{4}+\frac{\lambda_{2}}{4}\left(\varphi^{4}+\varphi^{* 4}\right)+\frac{\lambda_{3}}{4}|\varphi|^{2}\left(\varphi^{2}+\varphi^{* 2}\right) .
$$

The following natural assumptions are made: the mass parameters of the potential are small in comparison with the Hubble parameter during inflation $m \ll H_{I}$, the self-coupling constants $\lambda_{i}$ are of the order of the gauge coupling constant $\alpha$, and $m$ is in the range $10^{2}-10^{4}$ $\mathrm{GeV}$. The energy density of $\varphi$ at the inflationary stage is of the order $H_{I}^{4}$, hence

$$
\varphi_{o}^{\max } \sim H_{I} \lambda^{-1 / 4}, \quad \dot{\varphi}_{o}=\left(H_{I}\right)^{2}, \quad B_{0}=H_{I}^{3} .
$$

At the end of the inflationary stage $\varphi$ starts to oscillate around its equilibrium. Its amplitude decreases due to the Universe expansion and due to the particle creation processes, resultant from the coupling of the scalar field to fermions $g \varphi f_{1} f_{2}$, where $g^{2} / 4 \pi=\alpha_{G U T}$.

In the SFC baryogenesis model, $B$ contained in the condensate, can be reduced considerably due to particle creation at the BV stage $[17,32]$. Therefore, at the stage, when baryon violation is large, $B$ contained in $\varphi$ condensate, is reduced due to particle production.

Here we provide numerical account for the particle creation processes of $\varphi$. BV becomes negligible at small $\varphi$. B which survives until B-conservation epoch $t_{B}$, is transferred to fermions and thus the excess of matter over antimatter is produced.

We have provided a numerical analysis $[26,27,32,33]$ of the evolution of the real and imaginary components of $\varphi, \varphi(t)=x+i y$, and of $B(t)$ from the inflationary stage until $t_{B}$. We developed a computer program in Fortran 77 using 4th order Runge-Kutta method. The system of ordinary differential equations, corresponding to the equation of motion for the real and imaginary part of $\varphi$ and $B$ was solved calculating $\Omega$ at each step. The numerical analysis included around 100 sets of parameters in their natural ranges of values: $\alpha=10^{-3}-5 \times 10^{-2}, H_{I}=10^{7}-10^{12} \mathrm{GeV}, m=100-1000 \mathrm{GeV}, \lambda_{1}=10^{-3}-5 \times 10^{-2}$, $\lambda_{2,3}=10^{-4}-5 \times 10^{-2}$. All $H_{I}$ values considered in our calculations are in agreement with the observational constraint from Planck data, namely: $H_{I}<3.7 \times 10^{-5} M_{P l} /(8 \pi)^{1 / 2}$.

For each set of the SFC baryogenesis model parameters we have calculated the final $B$ contained in the condensate $\varphi(t)$ before its decay. The dependence of the produced B on the parameters of the models (namely $m, H_{I}, \lambda_{i}$ and $\alpha$ ) were revealed in our analysis.

The produced baryon asymmetry $\beta$ in SFC baryogenesis model depends on the generated baryon excess $B$, the reheating temperature of the universe $T_{R}$ and the value of the Hubble parameter at the end of inflation $H_{I}$. Namely:

$$
\beta \sim N_{B} / T_{R}^{3} \sim B T_{R} / H_{I}
$$

The values $T_{R}$ and $H_{I}$ depend on the kind of inflation and reheating models.

Hence, in the present work we calculated the baryon asymmetry of the Universe produced in the SFC baryogenesis model, using the available results on B for all studied ranges of model parameters from ref. [27] and for the considered different models of inflation and reheating. In the next section we present the results of our analysis for the generated baryon asymmetry $\beta$ in several inflationary models and different reheating scenarios.

\section{Baryon Asymmetry in Different Inflationary Models}

\subsection{Notes on Inflation}

The idea of an exponential inflationary stage in the early evolution of the Universe has been established as an extension to the standard cosmological model to resolve several conceptional problems of the standard cosmological model, among which homo- 
geneity, isotropy, flatness of the Universe and the predicted overabundance of magnetic monopoles [34].

Now there exist hundreds models of inflation (see for example the Encyclopedia Inflationaris collection $[35,36])$. Recently the inflation models were probed by the Planck data. Planck 2013, 2015, and 2018 releases have put strong constraints on several types of inflationary models.

Chronologically, the first realistic inflationary model was created by Starobinsky in 1980 [37]. The model is in a good agreement with Planck18 data. In 1981 Linde [38] and Albrecht and Steinhardt [39] independently proposed a new inflation or slow-roll inflation model, where inflation occurred by a scalar field rolling down a potential energy hill, instead of tunneling out of a false vacuum state, as in ref. [34].

In 1983 the chaotic inflationary model was proposed, which does not require an initial state of thermal equilibrium, supercooling and tunneling from the false vacuum. This class of inflationary models has a single monomial potential $[40,41]$ :

$$
V(\psi)=\lambda M_{\mathrm{pl}}^{4}\left(\frac{\psi}{M_{\mathrm{pl}}}\right)^{p},
$$

where inflation occurs at $\psi>M_{\mathrm{pl}}$. Planck18 data disfavors potentials with $p \geq 2$ but models with simple linear potentials $p=1$ or $p=2 / 3$ and fractional power monomials are more acceptable. Other popular inflationary model is the model of quintessential inflation of Peebles and Vilenkin [42], which provides a unified description for both the inflationary stage and the current acceleration stage of the Universe using a single scalar field potential. Some model improvements were proposed lately to obtain agreement with the recent Planck18 observational data [43].

Thus, recent Planck CMB data [44] put constraints on inflationary models.

We have considered here the following inflationary models: the new inflation [38,39], Shafi-Vilenkin model of new inflation, chaotic inflation $[40,41,45]$, Shafi-Vilenkin model of chaotic inflation, chaotic inflation in SUGRA, Starobinsky inflation [46], MSSM inflation and quintessential inflation.

\subsection{Notes on Reheating}

Moreover the great variety of inflationary models, there exist also different possibilities for reheating realizations after inflation. During reheating the inflaton energy is transferred to other dynamical degrees of freedom, which results in radiation dominated stage of the Universe. However, there exist different reheating mechanisms [4]. The resultant $T_{R}$ depends on the way reheating proceeds: namely reheating by perturbative and by nonperturbative decay of the inflaton $\psi$, it depends also on the inflaton decay rate, on the spectrum of inflaton decay particles, on the thermalization after inflation (instantaneous or delayed), etc. [47]. There exist $\mathrm{CMB}$ and BBN constraints on the inflationary reheating temperature $T_{R}$. Reheating should proceed before BBN and $T_{R}>5 \mathrm{MeV}$, so that low reheating temperature would not strongly affect the properties of neutrino and consequently He production during BBN and CMB characteristics [48-51].

On the other hand, reheating should proceed at low enough energy so that GUT symmetry is not restored and thus monopole problem is evaded. Moreover, in SUSY models the reheating temperature should be $T_{R}<10^{7}-10^{9} \mathrm{GeV}$ to avoid gravitino overproduction, which can destroy BBN predictions. This constraint holds in case gravitinos are in the mass range (100 GeV-1 TeV) [52]. When gravitino mass is $>10 \mathrm{TeV}$, another constraint holds: $T_{R}<10^{11} \mathrm{GeV}$, following from the constraints on gravitino number from overclosure bound $[53,54]$.

In the 1990s, preheating by perturbative decay of the inflaton $\psi$ into fermions was considered. See the pioneer works of Refs. [2,55]. Reheating takes place when $H$ drops to the value of $\Gamma$, the total decay rate of the inflaton, and inflaton decay becomes effective. Using the Friedmann equation and assuming an instantaneous conversion of the inflaton 
energy at the end of inflation into radiation $\rho_{r}=g_{*} \pi^{2} / 30 T^{4}=\rho_{\psi}$ and fast thermalization, the reheating temperature is given by:

$$
T_{R}=\left(90 / 8 \pi^{3} g_{*}\right)^{1 / 4}\left(M_{P l} H\right)^{1 / 2} .
$$

Reheating completes when $H$ becomes less than $\Gamma / 2$. Then an upper bound is obtained for $\Gamma=2 H$ in the case of efficient thermalization, namely:

$$
T_{R}=\left(90 / 32 \pi^{3} g_{*}\right)^{1 / 4}\left(M_{P l} \Gamma\right)^{1 / 2},
$$

where $g_{*}$ is of the order $10^{2}, T_{R} \sim 0.1\left(M_{P l} \Gamma\right)^{1 / 2}$. Then the typical $T_{R}$ is less than $10^{9}$ $\mathrm{GeV}[56,57]$.

However, $\psi$ may decay into other bosons due to broad resonance [56-59]. In this case $T_{R}$ may be $\sim 10^{12} \mathrm{GeV}$, i.e., much higher than $T_{R}$ estimated in Equation (13). Nonperturbative preheating was discussed for example in Refs. [60-63]. On the other hand, $T_{R}$ may be much smaller than these estimations in the case of slow thermalization when local thermodynamical equilibrium is not reached until the beginning of the RD epoch. This is usually the case for small inflaton couplings and very big inflaton masses. The conditions for efficient or inefficient thermalization were discussed in ref. [5].

In conclusion, there are numerous possibilities for reheating resulting in a wide range for the allowed values of $T_{R}$. We have used in our analysis $T_{R}$ in the range $\left[10^{5}, 10^{14}\right] \mathrm{GeV}$.

\subsection{Baryon Asymmetry in Different Inflationary Models-Results}

Here we present our results of the baryon asymmetry value calculated for different reheating possibilities and different inflationary scenarios. First consideration of SFC baryogenesis model in different inflationary scenarios and preliminary results were reported in ref. $[64,65]$. In the present work we considered all $B$-excess values in the whole range of studied parameter sets of the SFC baryogenesis model.

The results of our analysis on the calculated $\beta$ for certain $T_{R}$ values corresponding to different inflationary scenarios and different types of thermalization are presented below:

\subsubsection{Inflationary Models with Overproduction of Baryon Asymmetry}

In the case of new inflation $[39,66]$ for $H_{I}=10^{10} \mathrm{GeV}$ and $T_{R}=10^{14} \mathrm{GeV}$ we have found that the calculated baryon asymmetry for all sets of model parameters is several orders of magnitudes bigger than the observational value $\beta_{o b s}$. (The same holds if one varies $H_{I}$ within the range $\left[5 \times 10^{9}, 5 \times 10^{10}\right] \mathrm{GeV}$.)

In the new inflation model by Shafi and Vilenkin [67] for $H_{I}=3 \times 10^{9} \mathrm{GeV}$ and $T_{R}=3 \times 10^{7} \mathrm{GeV}$, the calculated baryon asymmetry again is much bigger than $\beta_{\text {obs }}$, namely $\beta>10^{-7}$. (We have calculated $\beta$ for all sets of the SFC model parameters and for $H_{I}$ in the range $\left[10^{8}-10^{10}\right] \mathrm{GeV}$.)

In the case of chaotic inflation, for $H_{I} \in\left[10^{11}, 10^{12}\right] \mathrm{GeV}$, and $T_{R}<3 \times 10^{14} \mathrm{GeV}$, namely we have used $T_{R} \in\left[10^{12}, 10^{14}\right] \mathrm{GeV}$, the calculated asymmetry is $\beta>10^{-5}$.

For the simplest Shafi-Vilenkin model in chaotic inflation $T_{R}=10^{12}-10^{13} \mathrm{GeV}$ again $\beta>10^{-7}$. We have provided the analysis for $H_{I} \in\left[5 \times 10^{9}, 10^{12}\right] \mathrm{GeV}$.

However, depending on the inflaton decay rate, decay channels, couplings, $T_{R}$ may be lower, namely $T_{R} \in\left[10^{8}, 10^{11}\right] \mathrm{GeV}$, thus allowing successful baryogenesis in these chaotic inflationary models, i.e., even in the case of big $H_{I} \sim 10^{12} \mathrm{GeV} \beta_{o b s}$ can be obtained (as will be discussed in the next subsection).

In MSSM inflation model $[68,69]$ with $H_{I}=1 \mathrm{GeV}, T_{R}=2 \times 10^{8} \mathrm{GeV}$, SCF baryogenesis model does not work, $\beta>>\beta_{o b s}$. Moreover, this inflationary model also has severe problems with gravitino overproduction, violating BBN and the observational indications for dark matter abundance. 
3.3.2. Inflationary Models with Successful Production of the Observed Baryon Asymmetry

We have used the values of $T_{R}$ and $m_{\psi}$ for $\alpha_{\psi}=10^{-11}$ from ref. [5] to calculate the baryon asymmetry for the different cases and different $T_{R}$. (Assuming $\Gamma=\alpha_{\psi} m_{\psi}$ the authors have calculated $T_{R}\left(m_{\psi}\right)$ from ref. [70] for different $\alpha_{\psi}$ and for delayed and efficient thermalization.)

In the case of efficient thermalization we have found that the production of the observed value of $\beta$ is possible for $H_{I}=10^{12} \mathrm{GeV}, T_{R}=6.2 \times 10^{9} \mathrm{GeV}$ and specific sets of SFC model's parameters, namely: $\lambda_{1}=5 \times 10^{-2}, \alpha \in\left[3 \times 10^{-2}, 5 \times 10^{-2}\right]$, $\lambda_{2}=\lambda_{3} \in\left[10^{-3}, 10^{-2}\right], m=350 \mathrm{GeV}$. For $H_{I}=10^{11} \mathrm{GeV}$ and $T_{R}=1.9 \times 10^{9} \mathrm{GeV}$ the production of the observed value of $\beta$ is possible when $\lambda_{1}=\alpha=5 \times 10^{-2}, \lambda_{2}=\lambda_{3}=10^{-2}$ and $m=100,200$ and $350 \mathrm{GeV}$.

In the case of delayed thermalization there appear possibilities for the production of $\beta_{\text {obs }}$, corresponding to $H_{I}=10^{12} \mathrm{GeV}, T_{R}=4.5 \times 10^{8} \mathrm{GeV}$ and several different sets of model parameters in the following ranges $\lambda_{1} \in\left[5 \times 10^{-2}, 10^{-2}\right] ; \alpha \in\left[5 \times 10^{-2}, 10^{-2}\right]$, $\lambda_{2}=\lambda_{3}=10^{-3}$ and $m=350-500 \mathrm{GeV}$. It could be easily seen that the strongest influence comes from $\alpha$ parameter of the model and then from $m$. For fine-tuning $\lambda_{1}$ can be used and then $\lambda_{2}=\lambda_{3}$ parameters of the SCF model. (We have studied $H_{I}$ in the range $\left[10^{7}-10^{12}\right]$ $\mathrm{GeV}$.)

For monomial potential of eqn. 8 we have calculated $\beta$ for $p=2 / 3$ and $T_{R} \in\left[10^{8}, 10^{11}\right]$ GeV. $\beta_{o b s}$ can be produced for $T_{R}=10^{9} \mathrm{GeV}$ and $H_{I} \sim 10^{11} \mathrm{GeV}$.

In the case of modified Starobinsky inflation [37] $T_{R}=0.1\left(\Gamma M_{P l}\right)^{1 / 2}=10^{9} \mathrm{GeV}$, $H_{I}=10^{11} \mathrm{GeV}$, successful baryogenesis is possible for the efficient thermalization as well. Namely $\beta=\beta_{\text {obs }}$ was found possible for several sets of model's parameters. (We have studied $H_{I}$ in the range $\left[5 \times 10^{6}, 10^{12}\right] \mathrm{GeV}$.) For the simplest extension of the Starobinsky inflation see ref. [71].

For chaotic inflation in SUGRA [72] with $T_{R}>10^{9} \mathrm{GeV}$ it is possible to generate $\beta_{\text {obs }}$.

In quintessential inflation with $T_{R}=2 \times 10^{5} \mathrm{GeV}$ and decay into massless particles, the production of the baryon asymmetry value is successful for $H_{I}=10^{12} \mathrm{GeV}$ and several sets of SCF model parameters as well. When $m=350 \mathrm{GeV}$, the favored value of $\alpha$ parameter is $\alpha=10^{-3}$ within a wide range of $\lambda_{1} \in\left[10^{-3}, 5 \times 10^{-2}\right]$ and $\lambda_{2}=\lambda_{3} \in\left[10^{-4}, 5 \times 10^{-3}\right]$. Again, strong dependence of $\alpha$ parameter may be mentioned since $\alpha$ reflects on the time of the scalar field decay and therefore it has strong influence on the baryon excess and baryon asymmetry values.

The particular parameters sets of the SCF baryogenesis model for which successful production of the baryon asymmetry close to its observational value is possible, corresponding to different inflationary models and different types of thermalization, are listed in Table 1. Fixing the inflationary model it is possible to fix the parameters of the SFC baryogenesis model.

Table 1. Successful production of the observed baryon asymmetry value $\beta_{o b s}$ for particular sets of SCF model parameters in different inflationary scenarios.

\begin{tabular}{|c|c|c|c|}
\hline Starobinsky Inflation & $H_{I}=10^{11} \mathrm{GeV} ; T_{R}=10^{9} \mathrm{GeV}$ & $\begin{array}{l}\lambda_{1}=\alpha=5 \times 10^{-2} \\
\lambda_{2}=\lambda_{3}=10^{-2}, m=100 \mathrm{GeV} \\
\beta=9.3 \times 10^{-10}\end{array}$ & \\
\hline & $H_{I}=10^{12} \mathrm{GeV} ; T_{R}=10^{9} \mathrm{GeV}$ & $\begin{array}{l}\lambda_{1}=5 \times 10^{-2}, \alpha=3 \times 10^{-2} \\
\lambda_{2}=\lambda_{3}=10^{-3}, m=350 \mathrm{GeV} \\
\beta=6.6 \times 10^{-10}\end{array}$ & $\begin{array}{l}\lambda_{1}=\alpha=5 \times 10^{-2}, \\
\lambda_{2}=\lambda_{3}=10^{-3}, m=350 \mathrm{GeV} \\
\beta=8.0 \times 10^{-10^{\prime}}\end{array}$ \\
\hline Quintessential Inflation & $H_{I}=10^{12} \mathrm{GeV} ; T_{R}=2 \times 10^{5} \mathrm{GeV}$ & $\begin{array}{l}\lambda_{1}=5 \times 10^{-3}, \alpha=10^{-3} \\
\lambda_{2}=\lambda_{3}=10^{-4}, m=350 \mathrm{GeV} \\
\beta=4.6 \times 10^{-10^{\prime}}\end{array}$ & $\begin{array}{l}\lambda_{1}=10^{-2}, \alpha=10^{-3} \\
\lambda_{2}=\lambda_{3}=10^{-4}, m=350 \mathrm{GeV} \\
\beta=7.8 \times 10^{-10^{\prime}}\end{array}$ \\
\hline $\begin{array}{l}\text { Chaotic Inflation, Efficient } \\
\text { Thermalization }\end{array}$ & $H_{I}=10^{12} \mathrm{GeV} ; T_{R}=6.2 \times 10^{9} \mathrm{GeV}$ & $\begin{array}{l}\lambda_{1}=\alpha=5 \times 10^{-2} \\
\lambda_{2}=\lambda_{3}=10^{-2}, m=350 \mathrm{GeV} \\
\beta=7.4 \times 10^{-10^{\prime}}\end{array}$ & \\
\hline $\begin{array}{l}\text { Chaotic Inflation, Delayed } \\
\text { Thermalization }\end{array}$ & $H_{I}=10^{12} \mathrm{GeV} ; T_{R}=4.5 \times 10^{8} \mathrm{GeV}$ & $\begin{array}{l}\lambda_{1}=\alpha=10^{-2}, \lambda_{2}=\lambda_{3}=10^{-3} \\
m=350 \mathrm{GeV}, \beta=9.5 \times 10^{-10}\end{array}$ & $\begin{array}{l}\lambda_{1}=\alpha=5 \times 10^{-2}, \\
\lambda_{2}=\lambda_{3}=10^{-3}, m=350 \mathrm{GeV} \\
\beta=3.6 \times 10^{-10^{\prime}}\end{array}$ \\
\hline
\end{tabular}


As it can be seen from Table 1 in the case of Starobinsky and chaotic inflationary scenarios with successful production of the baryon asymmetry value for $T_{R} \in[4.5 \times$ $\left.10^{8}, 6.2 \times 10^{9}\right] \mathrm{GeV}$ and $H_{I} \in\left[10^{11}, 10^{12}\right]$, the SFC parameters can be approximately fixed they lie within the following ranges: $m \sim 350 \mathrm{GeV}$ (with one exception) $\alpha \in\left[10^{-2}, 5 \times 10^{-2}\right]$, $\lambda_{1} \sim 5 \times 10^{-2}, \lambda_{2,3} \in\left[10^{-3}, 10^{-2}\right]$.

In the case of quintessential inflation, however, for $H_{I} \sim 10^{12} \mathrm{GeV}$ and $m \sim 350$ $\mathrm{GeV}$, the reheating temperature is much lower $T_{R} \sim 2 \times 10^{5} \mathrm{GeV}$ and the rest of the SFC parameters - the coupling constants have lower values, namely $\alpha \sim 10^{-3}, \lambda_{1} \in$ $\left[5 \times 10^{-3}, 10^{-2}\right], \lambda_{2,3} \in\left[10^{-4}, 5 \times 10^{-5}\right]$.

Quintessential inflationary models need the smallest parameters $\lambda_{i}$ and $\alpha$, by order of magnitude smaller than the ones in other inflationary models considered here. If the requirement for the value of $\alpha$ to be close to $\alpha_{G U T}$ is imposed, SFC baryogenesis cannot be realized in Quintessential inflation. Thus, from a fundamental physics viewpoint the Starobinski model and chaotic inflationary model seem more realistic.

In Figure 1 we present in the $\alpha-\lambda_{2,3}$ plane the inflationary models in which the closest to the observational baryon asymmetry value is generated. The other fixed values for model parameters are: $\lambda_{1}=5 \times 10^{-2}, m=350 \mathrm{GeV}$ and $H_{I}=10^{12} \mathrm{GeV}$. Note that, however, the models correspond to different reheating temperatures, as given in Table 1.

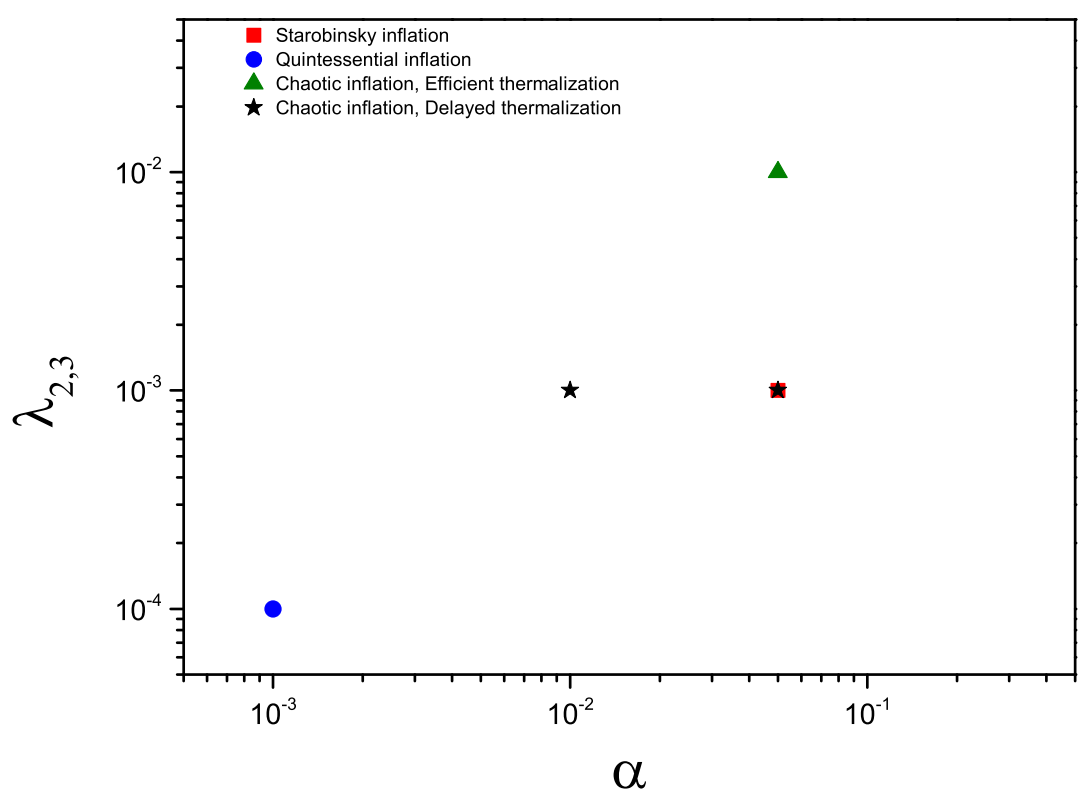

Figure 1. The figure presents different inflationary models in the $\alpha-\lambda_{2,3}$ plane for which successful SFC baryogenesis is achieved for the following parameters: $\lambda_{1}=5 \times 10^{-2}, m=350 \mathrm{GeV}$ and $H_{I}=10^{12} \mathrm{GeV}$.

We also have results for SUGRA inflationary model. In case of $T_{R} \sim 10^{12} \mathrm{GeV}$ the obtained $\beta$ are bigger than the observational value independent of the $H_{I}$ value assumed. For $T_{R}=10^{9} \mathrm{GeV}$, the results coincide with the ones for the Starobinsky inflationary model.

In this work we have not considered inflationary models, such as power law inflation, braneworld inflation, quintessential power law inflation, which require very high $H_{I}$ values, namely higher than $H_{I} \sim 10^{12} \mathrm{GeV}$, which was the upper limit used in our SFC baryogenesis model. To continue the analysis of SFC baryogenesis model for such types of inflationary models it is necessary to expand the numerical analysis towards higher values of $H_{I}$.

\section{Conclusions}

Based on the numerical analysis of the evolution of baryon charge $B(t)$ produced in the SFC baryogenesis model and the estimation of the produced baryon asymmetry 
$\beta$ for different sets of model parameters and different reheating temperatures of several inflationary scenarios we have shown that:

(i) SFC baryogenesis model produces baryon asymmetry by orders of magnitude bigger than the observed one for the following inflationary models: new inflation, new inflation model by Shafi and Vilenkin, chaotic inflation with high reheating temperature, the simplest Shafi-Vilenkin chaotic inflationary model and MSSM inflation.

For these models SCF baryogenesis needs strong diluting mechanisms to reduce the resultant baryon excess at low energies to its observational value today. (ii) SFC baryogenesis model produces similar to the observed baryon asymmetry value in the following inflationary models: Modified Starobinsky inflation, chaotic inflation with lower reheating temperature, chaotic inflation in SUGRA and Quintessential inflation. In case of delayed thermalization, when $T_{R}$ is much lower, a successful SFC baryogenesis may be achieved more easily in the chaotic inflationary models. Curiously enough among these are also models preferred by the Planck CMB data analysis.

It is intriguing that there is an overlap between the disfavored and favored inflationary models from our analysis based on successful SFC baryogenesis and the ones from Planck data. For comparison, the recent Planck data disfavors chaotic inflationary models with monomial potentials with power $\mathrm{p}$ bigger or equal to 2 and constrains low scale SUSY. It favors $R^{2}$ inflationary models (Starobinsky-like inflationary models) and inflationary models with monomial potential with $p<2$.

However, choosing from fundamental physics considerations the value of $\alpha$ to be the value closest to the $\alpha_{G U T}$, the number of realistic models reduces. Then Starobinski model and chaotic inflationary model are more realistic, while the quintessential inflation is not among the preferred models by SFC baryogenesis. The latter is in accord with the Planck data which supports constant dark-energy density - a cosmological constant, which at the present epoch dominates the energy density of the universe.

Vice versa, fixing the inflationary model we can fix the SFC model parameters which provide successful production of the observed baryon asymmetry.

Author Contributions: Conceptualization, D.K.; methodology, D.K.; software, M.P.; validation, D.K. and M.P.; formal analysis, M.P. and D.K.; writing — original draft preparation, D.K. and M.P.; writingreview and editing, D.K.; visualization M.P.; supervision, D.K. All authors have read and agreed to the published version of the manuscript.

Funding: This research received no external funding.

Institutional Review Board Statement: Not applicable.

Informed Consent Statement: Not applicable.

Conflicts of Interest: The authors declare no conflict of interest.

\section{References}

1. Linde, A. Prospects of Inflation. Phys. Scr. 2005, T117, 40-48. [CrossRef]

2. Dolgov, A.; Kirilova, D. On particle creation by a time dependent scalar field. Sov. J. Nucl. Phys. 1990, 51, $172-177$.

3. Felder, G.; Kofman, L.; Linde, A. Gravitational Particle Production and the Moduli Problem. JHEP 2000, 2, 27. [CrossRef]

4. Moghaddam, H. Reheating in the Early Universe Cosmology. Ph.D. Thesis, McGill University, Montreal, QC, Canada, 2017.

5. Mazumdar, A.; Zaldivar, B. Quantifying the reheating temperature of the universe. Nucl. Phys. 2014, B886, 312-327. [CrossRef]

6. Steigman, G. Observational Tests of Antimatter Cosmologies. Ann. Rev. Astron. Astrophys. 1976, 14, 339-372. [CrossRef]

7. Steigman, G. When clusters collide: Constraints on antimatter on the largest scales. J. Cosmol. Astropart. Phys. $2008,0910,001$. [CrossRef]

8. Stecker, F. On the nature of the baryon asymmetry. Nucl. Phys. B 1985, 252, 25-36. [CrossRef]

9. Ballmoos, P. Antimatter in the Universe: Constraints from gamma-ray astronomy. Hyperfine Interact. 2014, 228, 91-100. [CrossRef]

10. Dolgov, A. Antimatter in the universe and laboratory. EPJ Web Conf. 2015, 95, 03007. [CrossRef]

11. Pettini, M.; Cooke, R. A new, precise measurement of the primordial abundance of Deuterium. Mon. Not. R. Astron. Soc. 2012, 425, 2477-2486. [CrossRef]

12. Ade, P.; Aghanim, N.; Arnaud, M.; Ashdown, M.; Aumont, J.; Baccigalupi, C.; Banday, A.; Barreiro, R.; Bartlett, J.; Bartolo, N.; et.al. [Planck Collaboration], Planck 2015 results. Astron. Astrophys. 2016, 594, A13. 
13. Sakharov, A. Violation of CP Invariance, C asymmetry, and baryon asymmetry of the universe. JETP 1967, 5, 32-35.

14. Kuzmin, V.; Rubakov, V.; Shaposhnikov, M. Constraining Antimatter Domains in the Early Universe with Big Bang Nucleosynthesis. Phys. Rev. Lett. 1985, 84, 3756.

15. Fukugita, M.; Yanagida, T. Barygenesis without grand unification. Phys. Lett. B 1986, 174, 45-47. [CrossRef]

16. Affleck, I.; Dine, M. A New Mechanism for Baryogenesis. Nucl. Phys. B 1985, 249, 361-380. [CrossRef]

17. Dolgov, A.; Kirilova, D. Baryon charge condensate and baryogenesis. J. Mosc. Phys. Soc. 1991, 1, $217-229$.

18. Bastero-Gil, M.; Berera, A.; Ramos, R.; Rosa, J. Warm baryogenesis. Phys. Lett. B 2012, 712, 425-429. [CrossRef]

19. Davoudias, H.; Kitano, R.; Kribs, G.; Murayama, H.; Steinhardt, P. Gravitational Baryogenesis. Phys. Rev. Lett. $2004,93,201301$. [CrossRef]

20. Lambiase, G.; Scarpetta, G. Baryogenesis in $\mathrm{f}(\mathrm{R})$ theories of gravity. Phys. Rev. D 2006, 74, 087504. [CrossRef]

21. Sahoo, P.; Bhattacharjee, S. Gravitational Baryogenesis in Non-Minimal Coupled $\mathrm{f}(\mathrm{R}, \mathrm{T})$ Gravity. Int. J. Theor. Phys. 2020, 59, 1451-1459. [CrossRef]

22. Bhattacharjee, S.; Sahoo, P. Baryogenesis in $\mathrm{f}(\mathrm{Q}, \mathrm{T})$ gravity. Eur. Phys. J. C 2020, 80, 289. [CrossRef]

23. Chizhov, M.; Kirilova, D. Generation of the 128 h-1 Mpc periodicity of the universe in the scalar field condensate baryogenesis. AATr 1996, 10, 69-75.

24. Kirilova, D.; Chizhov, M. Non-GUT baryogenesis and large-scale structure of the Universe. MNRAS 2000, 314, 256-262. [CrossRef]

25. Kirilova, D. Baryogenesis model predicting antimatter in the Universe. Nucl. Phys. Proc. Suppl. 2003, 122, 404-408. [CrossRef]

26. Kirilova, D.; Panayotova, M. The dependence of the baryon asymmetry generation on the couplings of the baryon charge carrying scalar field. BAJ 2014, 20, 45-50.

27. Kirilova, D.; Panayotova, M. Parameterizing the SFC Baryogenesis Model. Adv. Astron. 2015, 465, 425342. [CrossRef]

28. Panayotova, M.; Kirilova, D. Baryogenesis with Scalar Field Condensate and Baryon Assymmetry of the Universe. Bulg. J. Phys. 2016, 43, 327-333.

29. Vilenkin, A.; Ford, L. Gravitational effects upon cosmological phase transitions. Phys. Rev. 1982, D26, 1231. [CrossRef]

30. Bunch, T.; Davies, P. Quantum field theory in de Sitter space: Renormalization by point-splitting. Proc. R. Soc. Lond. Ser. A 1978, $360,117$.

31. Starobinsky, A. Dynamics of phase transition in the new inflationary universe scenario and generation of perturbations. Phys. Lett. 1982, B117, 175-178. [CrossRef]

32. Kirilova, D.; Panayotova, M. The role of particle creation processes in the scalar condensate baryogenesis model. Bulg. J. Phys. 2007, 34, 330-335.

33. Kirilova, D.; Panayotova, M. Inhomogeneous baryogenesis model and antimatter in the Universe In Proceedings of the 8th Serbian-Bulgarian Astronomical Conference (VIII SBGAC), Leskovac, Serbia, 8-12 May 2012.

34. Guth, A. Inflationary universe: A possible solution to the horizon and flatness problems. Phys. Rev. D 1981, 23, 347. [CrossRef]

35. Martin, J.; Ringeval, C.; Vennin, V. Encyclopaedia Inflationaris. Phys. Dark Univ. 2014, 5-6, 75-235. [CrossRef]

36. Martin, J.; Ringeval, C.; Trotta, R.; Vennin, V. The Best Inflationary Models After Planck. JCAP03 2014, 1403, 039. [CrossRef]

37. Starobinsky, A. A new type of isotropic cosmological models without singularity. Phys. Lett. 1980, B91, 99-102. [CrossRef]

38. Linde, A. A new inflationary universe scenario: A possible solution of the horizon, flatness, homogeneity, isotropy and primordial monopole problems. Phys. Lett. 1982, B108, 389-393. [CrossRef]

39. Albrecht, A.; Steinhardt, P. Cosmology for Grand Unified Theories with Radiatively Induced Symmetry Breaking. Phys. Rev. Lett. 1982, 48, 1220. [CrossRef]

40. Linde, A. Chaotic inflation. Phys. Lett. 1983, 129B, 177-181. [CrossRef]

41. Linde, A. Initial conditions for inflation. Phys. Lett. 1985, 162B, 281-286. [CrossRef]

42. Peebles, P.; Vilenkin, A. Quintessential inflation. Phys. Rev. 1999, D59, 063505. [CrossRef]

43. Haro, J.; Amoros, J.; Pan, S. The Peebles and Vilenkin quintessential inflation model revisited. Eur. Phys. J. 2019, C79, 505. [CrossRef]

44. Akrami, Y.; Arroja, F.; Ashdown, M.; Aumont, J.; Baccigalupi, C.; Ballardini, M.; Banday, A.J.; Barreiro, R.B.; Bartolo, N.; Basak, S.; et al. [Plank Collaboration]. Planck 2018 results. X. Constraints on inflation. Astron. Astroph. 2020, 641, A10.

45. Linde, A. Particle Physics and Inflationary Cosmology; Harwood: Chur, Switzerland, 1990.

46. Kofman, L.; Linde, A.; Starobinsky, A. Inflationary universe generated by the combined action of a scalar field and gravitational vacuum polarization. Phys. Lett. B 1985, 157, 361-367. [CrossRef]

47. Marko, A.; Gasperis, G.; Paradis, G.; Cabella, P. Energy Density, Temperature and Entropy Dynamics in Perturbative Reheating. Phys. Rev. 2019, D100, 123532.

48. Martin, J.; Ringeval, C. First CMB Constraints on the Inflationary Reheating Temperature. Phys. Rev. 2010, D82, 023511. [CrossRef]

49. Martin, J.; Ringeval, C.; Vennin, V. Information Gain on Reheating: The One Bit Milestone. Phys. Rev. Lett. 2015, 114, 081303. [CrossRef] [PubMed]

50. Kawasaki, M.; Kohri, K.; Sugiyama, N. Cosmological Constraints on Late-time Entropy Production. Phys. Rev. Lett. 1999, 82, 4168. [CrossRef]

51. Salas, P.; Lattanzi, M.; Mangano, M.; Miele, G.; Pastor, S.; Pisanti, O. Bounds on very low reheating scenarios after Planck. PRD 2015, 92, 123534. [CrossRef] 
52. Kawasaki, M.; Kohri, K.; Moroi, T. Radiative decay of a massive particle and the nonthermal process in primordial nucleosynthesis. PRD 2001, 63, 103502. [CrossRef]

53. Giudice, G.; Tkachev, I.; Riotto, A. Non-Thermal Production of Dangerous Relics in the Early Universe. JHEP 1999, $9908,9$. [CrossRef]

54. Buchmuller, W. Thermal Production of Gravitinos. NPB 2001, 606, 518-544.

55. Traschen, J.; Brenberger, R. Particle production during out-of-equilibrium phase transitions. PRD 1990, 42, 2491. [CrossRef] [PubMed]

56. Kofman, L.; Linde, A.; Starobinski, A. Reheating after Inflation. Phys. Rev. Lett. 1994, 73, 3195. [CrossRef]

57. Kofman, L.; Linde, A.; Starobinski, A. Towards the Theory of Reheating After Inflation. Phys. Rev. D 1997, 56, 3258-3295. [CrossRef]

58. Boyanovsky, D.; D’Attanasio, M.; de Vega, H.J.; Holman, R.; Lee, D.-S. Linear versus nonlinear relaxation: Consequences for reheating and thermalization. PRD 1995, 52, 6805. [CrossRef]

59. Boyanovsky, D.; de Vega, H.; Holman, R.; S-Lee, D.; Singh, A. Dissipation via Particle Production in Scalar Field Theories. PRD 1995, 51, 4419-4444. [CrossRef]

60. Allahverdi, R.; Ferrantelli, A.; Garcia-Bellido, J; Mazumdar, A. Non-perturbative production of matter and rapid thermalization after MSSM inflation. Phys. Rev. 2011, D83, 123507.

61. Felder, G.; Kofman, L.; Linde, A. Instant Preheating. Phys. Rev. 1999, D59, 123523. [CrossRef]

62. Fujisaki, H.; Kumekawa, K.; Yamaguchi, M.; Yoshimura, M. Particle Production and Dissipative Cosmic Field. Phys. Rev. 1996, D53, 6805. [CrossRef]

63. Allahverdi, R.; Bastero-Gil, M.; Mazumdar, A. Is nonperturbative inflatino production during preheating a real threat to cosmology? Phys. Rev. 2001, D64, 023516. [CrossRef]

64. Kirilova, D.; Panayotova, M. Baryon asymmetry of the universe generated by scalar field condensate baryogenesis model in different inflationary scenarios. AIP Conf. Proc. 2019, 2075, 090017.

65. Kirilova, D.; Panayotova, M. Inflationary models, reheating and scalar field condensate baryogenesis. In Proceedings of the XII SB Astronomical Conference, Sokobanja, Serbia, 25-29 September 2020; pp. 39-47.

66. Dolgov, A.; Linde, A. Scalar field fluctuations in the expanding universe and the new inflationary universe scenario. Phys. Lett. 1982, 116B, 335-339.

67. Shafi, O.; Vilenkin, A. Inflation with SU(5). Phys. Rev. Lett. 1984, 52, 691. [CrossRef]

68. Allahverdi, A.; Enqvist, K.; Garcia-Bellido, J.; Mazumdar, A. Gauge-invariant inflaton in the minimal supersymmetric standard model. Phys. Rev. Lett. 2006, 97, 191304. [CrossRef]

69. Ferrantelli, A. Reheating, thermalization and non-thermal gravitino production in MSSM inflation. Eur. Phys. J. 2017, C77, 716. [CrossRef]

70. Abbott, L.; Fahri, E.; Wise, M. Particle production in the new inflationary cosmology. Phys. Lett. 1982, B117, 29-33. [CrossRef]

71. Van de Bruck, C.; Dansby, P.; Paduraru, L. Reheating and preheating in the simplest extension of Starobinsky inflation. Int. J. Mod. Phys. D 2013, 26, 13. [CrossRef]

72. Nanopoulos, D.; Olive, K.; Srednicki, M. After primordial inflation. Phys. Lett. 1983, B127, 30. [CrossRef] 